\title{
SOME REMARKS TO THE JACOBIAN CONJECTURE
}

\author{
Sylwia Lara-Dziembek, Grzegorz Biernat, Edyta Pawlak \\ Institute of Mathematics, Czestochowa University of Technology \\ Częstochowa, Poland \\ sylwia.lara@im.pcz.pl,grzegorz.biernat@im.pcz.pl,edyta.pawlak@im.pcz.pl
}

Received: 11 January 2017; accepted: 9 March 2017

\begin{abstract}
This work is related to the Jacobian Conjecture. It contains the formulas concerning algebraic dependence of the polynomial mappings having two zeros at infinity and the constant Jacobian. These relations mean that such mappings are non-invertible. They reduce the Jacobian Conjecture only to the case of mappings having one zero at infinity. This case is already solved by Abhyankar. The formulas presented in the paper were illustrated by the large example.
\end{abstract}

MSC 2010: $14 R 15$

Keywords: Jacobian, zero at infinity, Jacobian Conjecture

\section{Introduction}

In the paper [1], Abhyankar proved that the polynomial mapping $F: \mathbf{C}^{2} \rightarrow \mathbf{C}^{2}$ with a constant non-zero Jacobian has at most two zeros at infinity. This result was generalized in the paper [2]. Moreover, in the same paper [1], Abhyankar also proved that the Jacobian Conjecture [3-7] holds if and only if from the assumption that $J a c F=1$ it follows that the mapping $F$ not has two zeros at infinity.

It is not difficult to indicate an infinite family of polynomial non-invertible mapping having two zeros at infinity. These examples can be generalized. Therefore, in this work, we indicate explicit formulas that give the algebraic dependence of coordinates of polynomial mapping when its Jacobian is constant. We consider two groups of mappings, depending on the form of the leading forms of these mappings. Therefore these formulas adjudicate that there are no polynomial invertible mappings having two zeros at infinity.

\section{Algebraic dependence of polynomial mappings}

Let $f_{i}, h_{j}$ be the complex forms of variables $X, Y$ of degrees $i, j$ respectively and $i, j \geq 1$. 
Remark 1. Let

$$
f=(X Y)^{p}+f_{2 p-1}+f_{2 p-2}+f_{2 p-3}+\ldots+f_{1}
$$

and

$$
h=(X Y)^{q}+h_{2 q-1}+h_{2 q-2}+h_{2 q-3}+\ldots+h_{1}
$$

where $p \geq q \geq 1$.

If $\operatorname{Jac}(f, h)=$ const $=\operatorname{Jac}\left(f_{1}, h_{1}\right)$ then

$$
f=\left(X Y+\frac{1}{q} h_{2 q-1 \mid 1}\right)^{p}+A_{p-1}\left(X Y+\frac{1}{q} h_{2 q-1 \mid 1}\right)^{p-1}+\ldots+A_{1}\left(X Y+\frac{1}{q} h_{2 q-1 \mid 1}\right)
$$

and

$$
h=\left(X Y+\frac{1}{q} h_{2 k-1 \mid 1}\right)^{q}+B_{q-1}\left(X Y+\frac{1}{q} h_{2 q-1 \mid 1}\right)^{q-1}+\ldots+B_{1}\left(X Y+\frac{1}{q} h_{2 q-1 \mid 1}\right)
$$

for some constants $A_{1}, \ldots, A_{p-1}, B_{1}, \ldots, B_{q-1}$. The form $h_{2 q-1 \mid 1}$ is defined by the formula $h_{2 q-1}=X^{k-1} Y^{k-1} h_{2 q-1 \mid}$.

Remark 2. Let

$$
f=\left(X^{k} Y^{l}\right)^{p}+f_{(k+l) p-1}+f_{(k+l) p-2}+\ldots+f_{(k+l)(p-1)+1}+\ldots+f_{1}
$$

and

$$
h=\left(X^{k} Y^{l}\right)^{q}+h_{(k+l) q-1}+h_{(k+l) q-2}+\ldots+h_{(k+l)(q-1)+1}+\ldots+h_{1}
$$

where $k>l$ ( $k$ and $l$ are relativity prim) and $p \geq q \geq 1$.

If $\operatorname{Jac}(f, h)=$ const $=\operatorname{Jac}\left(f_{1}, h_{1}\right)$ then exist the forms $\hat{h}_{k+l-2}, \ldots, \hat{h}_{1}$ for which

$$
\begin{aligned}
& f=\left(X^{k} Y^{l}+\frac{1}{q} h_{(k+l) q-1 \mid k+l-1}+\frac{1}{q} \hat{h}_{k+l-2}+\ldots+\frac{1}{q} \hat{h}_{1}\right)^{p}+ \\
& +A_{p-1}\left(X^{k} Y^{l}+\frac{1}{q} h_{(k+l) q-1 \mid k+l-1}+\frac{1}{q} \hat{h}_{k+l-2}+\ldots+\frac{1}{q} \hat{h}_{1}\right)^{p-1}+\ldots+ \\
& +A_{1}\left(X^{k} Y^{l}+\frac{1}{q} h_{(k+l) q-1 \mid k+l-1}+\frac{1}{q} \hat{h}_{k+l-2}+\ldots+\frac{1}{q} \hat{h}_{1}\right)
\end{aligned}
$$


and

$$
\begin{aligned}
& h=\left(X^{k} Y^{l}+\frac{1}{q} h_{(k+l) q-1 \mid k+l-1}+\frac{1}{q} \hat{h}_{k+l-2}+\ldots+\frac{1}{q} \hat{h}_{1}\right)^{q}+ \\
& +B_{q-1}\left(X^{k} Y^{l}+\frac{1}{q} h_{(k+l) q-1 \mid k+l-1}+\frac{1}{q} \hat{h}_{k+l-2}+\ldots+\frac{1}{q} \hat{h}_{1}\right)^{q-1}+\ldots+ \\
& +B_{1}\left(X^{k} Y^{l}+\frac{1}{q} h_{(k+l) q-1 \mid k+l-1}+\frac{1}{q} \hat{h}_{k+l-2}+\ldots+\frac{1}{q} \hat{h}_{1}\right)
\end{aligned}
$$

for some constants $A_{1}, \ldots, A_{p-1}, B_{1}, \ldots, B_{q-1}$. The form $h_{(k+l) q-1 \mid k+l-1}$ of degree $k+l-1$ is defined by the formula $h_{(k+l) q-1}=\left(X^{k} Y^{l}\right)^{q-1} h_{(k+l) q-1 \mid k+l-1}$.

The authors try to place the proofs of the above hypotheses in the next article.

Corollary. Obviously, in all of these possible cases, the polynomials $f, h$ are algebraically dependent and so $\operatorname{Jac}(f, h)=0$.

The following example is the illustration of remark 2 .

Example. Let

$$
\begin{gathered}
f=\left(X^{2} Y\right)^{3}+\left.f_{8}\right|^{1)}+\left.f_{7}\right|^{2)}+\left.f_{6}\right|^{3)}+\left.f_{5}\right|^{4)}+\left.f_{4}\right|^{5)}+\left.f_{3}\right|^{6)}+\left.f_{2}\right|^{7)}+\left.f_{1}\right|^{8)} \\
h=\left(X^{2} Y\right)^{2}+\left.h_{5}\right|^{1)}+\left.h_{4}\right|^{2)}+\left.h_{3}\right|^{3)}+\left.h_{2}\right|^{4)}+\left.h_{1}\right|^{5)}+\left.0\right|^{(6)}+\left.0\right|^{7)}+\left.0\right|^{8)}
\end{gathered}
$$

Since the Jacobian is constant we have consecutively

$$
\begin{gathered}
\text { 1) } \operatorname{Jac}\left(\left(X^{2} Y\right)^{3}, h_{5}\right)=\operatorname{Jac}\left(\left(X^{2} Y\right)^{2}, f_{8}\right) \\
3\left(X^{2} Y\right)^{2} \operatorname{Jac}\left(X^{2} Y, h_{5}\right)=2 X^{2} Y \operatorname{Jac}\left(X^{2} Y, f_{8}\right)
\end{gathered}
$$

so

$$
\frac{3}{2} X^{2} Y \operatorname{Jac}\left(X^{2} Y, h_{5}\right)=\operatorname{Jac}\left(X^{2} Y, f_{8}\right)
$$

and appears

$$
\frac{3}{2} X^{2} Y h_{5}=f_{8}
$$




$$
\text { 2) } \operatorname{Jac}\left(\left(X^{2} Y\right)^{3}, h_{4}\right)+\underbrace{\operatorname{Jac}\left(f_{8}, h_{5}\right)}_{1^{\circ}}=\operatorname{Jac}\left(\left(X^{2} Y\right)^{2}, f_{7}\right)
$$

where

$$
1^{\circ}=\underbrace{\operatorname{Jac}\left(f_{8}, h_{5}\right)}_{1^{\circ}}=\operatorname{Jac}\left(\frac{3}{2} X^{2} Y h_{5}, h_{5}\right)=\frac{3}{2} h_{5} \operatorname{Jac}\left(X^{2} Y, h_{5}\right)
$$

therefore

$$
3\left(X^{2} Y\right)^{2} \operatorname{Jac}\left(X^{2} Y, h_{4}\right)+\frac{3}{2} h_{5} \operatorname{Jac}\left(X^{2} Y, h_{5}\right)=2 X^{2} Y \operatorname{Jac}\left(X^{2} Y, f_{7}\right)
$$

and

$$
3\left(X^{2} Y\right)^{2} h_{4}+\frac{3}{4} h_{5}^{2}=2 X^{2} Y f_{7}
$$

Thus $X^{2} Y$ divides $h_{5}^{2}$. We assume further that

$$
h_{5}=X^{2} Y h_{5 \mid 2}
$$

which implies

$$
f_{8}=\frac{3}{2}\left(X^{2} Y\right)^{2} h_{5 \mid 2}
$$

returning to equality (18) we obtain

$$
3\left(X^{2} Y\right)^{2} h_{4}+\frac{3}{4}\left(X^{2} Y\right)^{2} h_{5 \mid 2}^{2}=2 X^{2} Y f_{7}
$$

and occurs

$$
\begin{gathered}
\frac{3}{2} X^{2} Y h_{4}+\frac{3}{8} X^{2} Y h_{5 / 2}^{2}=f_{7} \\
\text { 3) } \operatorname{Jac}\left(\left(X^{2} Y\right)^{3}, h_{3}\right)+\underbrace{\operatorname{Jac}\left(f_{8}, h_{4}\right)}_{1^{\circ}}+\underbrace{\operatorname{Jac}\left(f_{7}, h_{5}\right)}_{2^{\circ}}=\operatorname{Jac}\left(\left(X^{2} Y\right)^{3}, f_{6}\right)
\end{gathered}
$$

where 


$$
\begin{aligned}
1^{\circ} & =\operatorname{Jac}\left(f_{8}, h_{4}\right)=\operatorname{Jac}\left(\frac{3}{2}\left(X^{2} Y\right)^{2} h_{5 \mid 2}, h_{4}\right)=\frac{3}{2} \operatorname{Jac}\left(\left(X^{2} Y\right)^{2} h_{5 \mid 2}, h_{4}\right)= \\
& =\frac{3}{2}\left(\left(X^{2} Y\right)^{2} \operatorname{Jac}\left(h_{5 \mid 2}, h_{4}\right)+h_{5 \mid 2} \operatorname{Jac}\left(\left(X^{2} Y\right)^{2}, h_{4}\right)\right)= \\
& =\frac{3}{2}\left(X^{2} Y\right)^{2} \mathrm{Jac}\left(h_{5 \mid 2}, h_{4}\right)+3 X^{2} Y h_{5 \mid 2} \mathrm{Jac}\left(X^{2} Y, h_{4}\right)
\end{aligned}
$$

and

$$
\begin{aligned}
& 2^{\circ}=\operatorname{Jac}\left(f_{7}, h_{5}\right)=\operatorname{Jac}\left(\frac{3}{2} X^{2} Y h_{4}+\frac{3}{8} X^{2} Y h_{5 \mid 2}^{2}, X^{2} Y h_{5 \mid 2}\right)= \\
& =\frac{3}{2} \operatorname{Jac}\left(X^{2} Y h_{4}, X^{2} Y h_{5 \mid 2}\right)+\frac{3}{8} \operatorname{Jac}\left(X^{2} Y h_{5 \mid 2}^{2}, X^{2} Y h_{5 \mid 2}\right)= \\
& =\frac{3}{2}\left(X^{2} Y h_{4} \operatorname{Jac}\left(X^{2} Y, h_{5 \mid 2}\right)-X^{2} Y h_{5 \mid 2} \operatorname{Jac}\left(X^{2} Y, h_{4}\right)+\left(X^{2} Y\right)^{2} \operatorname{Jac}\left(h_{4}, h_{5 \mid 2}\right)\right) \\
& +\frac{3}{8} X^{2} Y h_{5 \mid 2} \operatorname{Jac}\left(h_{5 \mid 2}, X^{2} Y h_{5 \mid 2}\right)= \\
& =\frac{3}{2} X^{2} Y h_{4} \operatorname{Jac}\left(X^{2} Y, h_{5 \mid 2}\right)-\frac{3}{2} X^{2} Y h_{5 \mid 2} \operatorname{Jac}\left(X^{2} Y, h_{4}\right) \\
& +\frac{3}{2}\left(X^{2} Y\right)^{2} \operatorname{Jac}\left(h_{4}, h_{5 \mid 2}\right)-\frac{3}{8} X^{2} Y h_{5 \mid 2}^{2} \operatorname{Jac}\left(X^{2} Y, h_{5 \mid 2}\right)
\end{aligned}
$$

Of the equation (29) results

$$
\begin{aligned}
& 3\left(X^{2} Y\right)^{2} \mathrm{Jac}\left(X^{2} Y, h_{3}\right)+\frac{3}{2} X^{2} Y \mathrm{Jac}\left(X^{2} Y, h_{5 \mid 2} h_{4}\right) \\
& +\frac{3}{8} X^{2} Y h_{5 \mid 2}^{2} \operatorname{Jac}\left(X^{2} Y, h_{5 \mid 2}\right)
\end{aligned}
$$

so

$$
3\left(X^{2} Y\right)^{2} h_{3}+\frac{3}{2} X^{2} Y h_{5 \mid 2} h_{4}+\frac{1}{8} X^{2} Y h_{5 \mid 2}^{3}+2 a_{6}\left(X^{2} Y\right)^{3}=2 X^{2} Y f_{6}
$$

and finally

$$
\frac{3}{2} X^{2} Y h_{3}+\frac{3}{4} h_{5 \mid 2} h_{4}+\frac{1}{16} h_{5 \mid 2}^{3}+a_{6}\left(X^{2} Y\right)^{2}=f_{6}
$$

4) $\operatorname{Jac}\left(\left(X^{2} Y\right)^{3}, h_{2}\right)+\underbrace{\operatorname{Jac}\left(f_{8}, h_{3}\right)}_{1^{\circ}}+\underbrace{\operatorname{Jac}\left(f_{7}, h_{4}\right)}_{2^{\circ}}+\underbrace{\operatorname{Jac}\left(f_{6}, h_{5}\right)}_{3^{\circ}}=\operatorname{Jac}\left(\left(X^{2} Y\right)^{3}, f_{5}\right)(29)$

Now we have 


$$
\begin{aligned}
1^{\circ} & =\operatorname{Jac}\left(f_{8}, h_{3}\right)=\operatorname{Jac}\left(\frac{3}{2}\left(X^{2} Y\right)^{2} h_{5 \mid 2}, h_{3}\right)=\frac{3}{2} \operatorname{Jac}\left(\left(X^{2} Y\right)^{2} h_{5 \mid 2}, h_{3}\right)= \\
& =\frac{3}{2}\left(\left(X^{2} Y\right)^{2} \operatorname{Jac}\left(h_{5 \mid 2}, h_{3}\right)+h_{5 \mid 2} \mathrm{Jac}\left(\left(X^{2} Y\right)^{2}, h_{3}\right)\right)= \\
& =\frac{3}{2}\left(X^{2} Y\right)^{2} \mathrm{Jac}\left(h_{5 \mid 2}, h_{3}\right)+3 X^{2} Y h_{5 \mid 2} \mathrm{Jac}\left(X^{2} Y, h_{3}\right)
\end{aligned}
$$

Next

$$
\begin{aligned}
2^{\circ} & =\operatorname{Jac}\left(f_{7}, h_{4}\right)=\operatorname{Jac}\left(\frac{3}{2} X^{2} Y h_{4}+\frac{3}{8} X^{2} Y h_{5 \mid 2}^{2}, h_{4}\right)= \\
& =\frac{3}{2} \operatorname{Jac}\left(X^{2} Y h_{4}, h_{4}\right)+\frac{3}{8} \operatorname{Jac}\left(X^{2} Y h_{5 \mid 2}^{2}, h_{4}\right)= \\
& =\frac{3}{2} h_{4} X^{2} Y \mathrm{Jac}\left(X^{2} Y, h_{4}\right)+\frac{3}{8}\left(X^{2} Y \mathrm{Jac}\left(h_{5 \mid 2}^{2}, h_{4}\right)+h_{5 \mid 2}^{2} \operatorname{Jac}\left(X^{2} Y, h_{4}\right)\right)= \\
& =\frac{3}{2} h_{4} X^{2} Y \mathrm{Jac}\left(X^{2} Y, h_{4}\right)+\frac{3}{4} X^{2} Y h_{5 \mid 2} \operatorname{Jac}\left(h_{5 \mid 2}, h_{4}\right)+\frac{3}{8} h_{5 \mid 2}^{2} \operatorname{Jac}\left(X^{2} Y, h_{4}\right)
\end{aligned}
$$

and

$$
\begin{aligned}
& 3^{\circ}=\operatorname{Jac}\left(f_{6}, h_{5}\right)=\operatorname{Jac}\left(\frac{3}{2} X^{2} Y h_{3}+\frac{3}{4} h_{5 \mid 2} h_{4}+\frac{1}{16} h_{5 \mid 2}^{3} h_{4}+a_{6}\left(X^{2} Y\right)^{2}, X^{2} Y h_{5 \mid 2}\right)= \\
& =\frac{3}{2} \operatorname{Jac}\left(X^{2} Y h_{3}, X^{2} Y h_{5 \mid 2}\right)-\frac{3}{4} \operatorname{Jac}\left(X^{2} Y h_{5 \mid 2}, h_{5 \mid 2} h_{4}\right)-\frac{1}{16} \operatorname{Jac}\left(X^{2} Y h_{5 \mid 2}, h_{5 \mid 2}^{3}\right) \\
& +2 a_{6}\left(X^{2} Y\right)^{2} \operatorname{Jac}\left(X^{2} Y, h_{5 \mid 2}\right)= \\
& =\frac{3}{2}\left(X^{2} Y h_{3} \operatorname{Jac}\left(X^{2} Y, h_{5 \mid 2}\right)-X^{2} Y h_{5 \mid 2} \operatorname{Jac}\left(X^{2} Y, h_{3}\right)+\left(X^{2} Y\right)^{2} \operatorname{Jac}\left(X^{2} Y h_{3}, h_{5 \mid 2}\right)\right) \\
& -\frac{3}{4}\left(X^{2} Y \operatorname{Jac}\left(h_{5 \mid 2}, h_{5 \mid 2} h_{4}\right)+h_{5 \mid 2} \operatorname{Jac}\left(X^{2} Y, h_{5 \mid 2} h_{4}\right)\right) \\
& -\frac{3}{16} h_{5 \mid 2}^{2} \operatorname{Jac}\left(X^{2} Y h_{5 \mid 2}, h_{5 \mid 2}\right)+2 a_{6}\left(X^{2} Y\right)^{2} \mathrm{Jac}\left(X^{2} Y, h_{5 \mid 2}\right)= \\
& =\frac{3}{2} X^{2} Y h_{3} \operatorname{Jac}\left(X^{2} Y, h_{5 \mid 2}\right)-\frac{3}{2} X^{2} Y h_{5 \mid 2} \operatorname{Jac}\left(X^{2} Y, h_{3}\right) \\
& +\frac{3}{2}\left(X^{2} Y\right)^{2} \operatorname{Jac}\left(X^{2} Y h_{3}, h_{5 \mid 2}\right)-\frac{3}{4} X^{2} Y h_{5 \mid 2} \operatorname{Jac}\left(h_{5 \mid 2}, h_{4}\right) \\
& -\frac{3}{4} h_{5 \mid 2}\left(h_{5 \mid 2} \operatorname{Jac}\left(X^{2} Y, h_{4}\right)+h_{4} \operatorname{Jac}\left(X^{2} Y, h_{5 \mid 2}\right)\right) \\
& -\frac{3}{16} h_{5 \mid 2}^{3} \operatorname{Jac}\left(X^{2} Y, h_{5 \mid 2}\right)+2 a_{6}\left(X^{2} Y\right)^{2} \operatorname{Jac}\left(X^{2} Y, h_{5 \mid 2}\right)
\end{aligned}
$$


Of the equation (29) results

$$
\begin{aligned}
& 3\left(X^{2} Y\right)^{2} \operatorname{Jac}\left(X^{2} Y, h_{2}\right)+\frac{3}{2} X^{2} Y \operatorname{Jac}\left(X^{2} Y, h_{5 \mid 2} h_{3}\right)+\frac{3}{2} h_{4} \operatorname{Jac}\left(X^{2} Y, h_{4}\right) \\
& -\frac{3}{8} h_{5 \mid 2}^{2} \operatorname{Jac}\left(X^{2} Y, h_{4}\right)-\frac{3}{4} h_{5 \mid 2} h_{4} \operatorname{Jac}\left(X^{2} Y, h_{5 \mid 2}\right)+\frac{3}{16} h_{5 \mid 2}^{3} \operatorname{Jac}\left(X^{2} Y, h_{5 \mid 2}\right) \\
& +2 a_{6}\left(X^{2} Y\right)^{2} \operatorname{Jac}\left(X^{2} Y, h_{5 \mid 2}\right)=2 X^{2} Y \operatorname{Jac}\left(X^{2} Y, f_{5}\right)
\end{aligned}
$$

hence

$$
\begin{aligned}
& 3\left(X^{2} Y\right)^{2} h_{2}+\frac{3}{2} X^{2} Y h_{5 \mid 2} h_{3}+\frac{3}{4} h_{4}^{2}-\frac{3}{8} h_{5 \mid 2}^{2} h_{4}+\frac{3}{16 \cdot 4} h_{5 \mid 2}^{4}+2 a_{6}\left(X^{2} Y\right)^{2} h_{5 \mid 2}= \\
& =2 X^{2} Y f_{5}
\end{aligned}
$$

and

$$
3\left(X^{2} Y\right)^{2} h_{2}+\frac{3}{2} X^{2} Y h_{5 \mid 2} h_{3}+\frac{3}{4}\left(h_{4}-\frac{1}{4} h_{5 \mid 2}^{2}\right)^{2}+2 a_{6}\left(X^{2} Y\right)^{2} h_{5 \mid 2}=2 X^{2} Y f_{5}
$$

Thus $X^{2} Y$ divides $\left(h_{4}-\frac{1}{4} h_{5 \mid 2}^{2}\right)^{2}$. We assume again that

$$
h_{4}-\frac{1}{4} h_{5 / 2}^{2}=X^{2} Y \hat{h}_{1}
$$

Therefore

$$
h_{4}=\frac{1}{4} h_{5 \mid 2}^{2}+X^{2} Y \hat{h}_{1}
$$

From the equation (35) we obtain

$$
3 X^{2} Y h_{2}+\frac{3}{4} h_{5 \mid 2} h_{3}+\frac{3}{8} X^{2} Y \hat{h}_{1}^{2}+a_{6} X^{2} Y h_{5 \mid 2}=f_{5}
$$

The equality (36) defines the function $\hat{h}_{1}$ which appears in remark 3 . This means that the polynomials $f$ and $h$ will be the form

$f=\left(X^{2} Y+\frac{1}{2} h_{5 \mid 2}+\frac{1}{2} \hat{h}_{1}\right)^{3}+A_{2}\left(X^{2} Y+\frac{1}{2} h_{5 \mid 2}+\frac{1}{2} \hat{h}_{1}\right)^{2}+A_{1}\left(X^{2} Y+\frac{1}{2} h_{5 \mid 2}+\frac{1}{2} \hat{h}_{1}\right)$

and

$$
h=\left(X^{2} Y+\frac{1}{2} h_{5 \mid 2}+\frac{1}{2} \hat{h}_{1}\right)^{2}+B_{1}\left(X^{2} Y+\frac{1}{2} h_{5 \mid 2}+\frac{1}{2} \hat{h}_{1}\right)
$$


Consequently

$$
\begin{gathered}
h_{3}=\frac{1}{2} h_{5 \mid 2} \hat{h}_{1}+B_{1} X^{2} Y \\
h_{2}=\frac{1}{4} \hat{h}_{1}^{2}+\frac{1}{2} B_{1} h_{5 \mid 2}
\end{gathered}
$$

and

$$
h_{1}=\frac{1}{2} B_{1} \hat{h}_{1}
$$

Similarly

$$
\begin{gathered}
f_{7}=\frac{3}{4} X^{2} Y h_{5 \mid 2}^{2}+\frac{3}{2}\left(X^{2} Y\right)^{2} \hat{h}_{1} \\
f_{6}=\frac{1}{8} h_{5 \mid 2}^{3}+\frac{3}{2} X^{2} Y h_{5 \mid 2} \hat{h}_{1}+A_{2}\left(X^{2} Y\right)^{2} \\
f_{5}=\frac{3}{4} X^{2} Y \hat{h}_{1}^{2}+\frac{3}{8} h_{5 \mid 2}^{2} \hat{h}_{1}+A_{2} X^{2} Y h_{5 \mid 2} \\
f_{4}=\frac{3}{8} h_{5 \mid 2} \hat{h}_{1}^{2}+A_{2} X^{2} Y \hat{h}_{1}+\frac{1}{4} A_{2} h_{5 \mid 2}^{2} \\
f_{3}=\frac{1}{8} \hat{h}_{1}^{3}+\frac{1}{2} A_{2} h_{5 \mid 2} \hat{h}_{1}+A_{1} X^{2} Y \\
f_{2}=\frac{1}{4} A_{2} \hat{h}_{1}^{2}+\frac{1}{2} A_{1} h_{5 \mid 2}
\end{gathered}
$$

and

$$
f_{1}=\frac{1}{2} A_{1} \hat{h}_{1}
$$

\section{Conclusions}

These hypotheses, tested by many examples, allow one to state that the polynomial mapping $(f, h): \mathbb{C}^{2} \rightarrow \mathbb{C}^{2}$ having two zeros at infinity are non-invertible 
(in the global sense). Therefore it remains necessary to analyze only the case when the mapping $(f, h)$ has only one zero at infinity and thus takes the form

$$
f=X^{p}+f_{p-1}+f_{p-2}+\ldots+f_{1}
$$

and

$$
h=X^{q}+h_{q-1}+h_{q-2}+\ldots+h_{1}
$$

where $f_{i}, h_{j}$ are the forms (of two complex variables) of degrees $i, j$ respectively.

We show that there are non-trivial class of mappings having one zero at infinity with the constant Jacobian, for which that Jacobian vanishes. It appears, therefore, that in the general case, the polynomial mapping having one zero at infinity and the constant Jacobian, must make the Jacobian vanish. This would mean that the Jacobian Conjecture takes place only in the case, when (cf. [1])

$$
f=A_{0} h^{n}+A_{1} h^{n-1}+\ldots+A_{n-1} h+a_{1} X, \quad n \geq 1, \quad A_{0} \neq 0, \quad a_{1} \neq 0
$$

and

$$
h=B_{0} X^{q}+B_{1} X^{q-1}+\ldots+B_{q-2} X^{2}+h_{1}, \quad q \geq 2, \quad B_{0} \neq 0, \quad \frac{\partial h_{1}}{\partial Y} \neq 0
$$

and also in the simplest case

$$
f=A_{0} X^{n}+A_{1} X^{n-1}+\ldots+A_{n-2} X^{2}+A_{n-1} X-c Y, \quad n \geq 2, \quad A_{0} \neq 0, \quad c \neq 0
$$

and

$$
h=B_{0} X, \quad B_{0} \neq 0
$$

The polynomials $f, h$ have in each case one zero at infinity and do not have the constant components as well as $\operatorname{deg} f \geq \operatorname{deg} h$.

\section{References}

[1] Abhyankar S.S., Expansion techniques in algebraic geometry, Tata Inst. Fundamental Research, Bombay 1977.

[2] Charzyński Z., Chądzyński J., Skibinski P., A Contribution to Keller's Jacobian Conjecture, Lecture Notes In Math. 1165, Springer-Verlag, Berlin, Heidelberg, New York 1985, 36-51.

[3] Wright D., On the Jacobian conjecture, Illinois J. Math. 1981, 25, 3, 423-440. 
[4] Van den Essen A., Polynomial automorphisms and the Jacobian conjecture, Progress in Matematics 190, Birkhäuser Verlag, Basel 2000.

[5] Bass H., Connell E.H., Wright D., The Jacobian conjecture: reduction of degree and formal expansion of the inverse, American Mathematical Society. Bulletin. New Series 1982, 7(2), 287-330.

[6] Fulghesu D., An Introduction to the Jacobian Conjecture, Minnesota State University Moorhead, October 12, 2010.

[7] Drużkowski L.M., The Jacobian Conjecture, Uniwersytet Jagielloński, Kraków 1993. 